\title{
Optical tunable delay lines using fiber ring with acousto-optic frequency shifters and EDFAs: I. experimental demonstration
}

\author{
Katsumi Takano $^{1 \mathrm{a})}$, Kiyoshi Nakagawa ${ }^{1}$, and Hiromasa Ito ${ }^{2}$ \\ ${ }^{1}$ Faculty of Engineering, Yamagata University \\ 4-3-16 Jonan, Yonezawa, Yamagata 992-8510, Japan \\ ${ }^{2}$ Research Institute of Electrical Communication, Tohoku University \\ 2-1-1 Katahira, Sendai 980-8577, Japan \\ a)ktakano@ieee.org
}

\begin{abstract}
Optical buffers are key components in optical packet switching network systems that prevent packet collision along the same virtual path and wait for header processing time. Conventional fiber loops for optical buffers present the drawback of unexpected oscillation at gain peak wavelength of the fiber loop. In this paper, an optical tunable delay line using a fiber ring with an acousto-optic frequency shifter and an erbium-doped fiber amplifier (EDFA) are demonstrated experimentally. The frequency shifter plays the role of preventing unexpected oscillation. Clear eye heights are visible in data packets through the delay line.
\end{abstract}

Keywords: optical communications, packet, buffer, delay line, EDFA, frequency shift

Classification: Photonics devices, circuits, and systems

\section{References}

[1] D. K. Hunter and I. Andonovic, "Approaches to optical internet packet switching," IEEE Commun. Mag., pp. 116-122, Sept. 2000.

[2] R. W. Boyd, "Slow light in bulk materials and optical fibers," Proc. OFC2006, Anaheim, USA, OTuA1, March 2006.

[3] D. Hunter, W. D. Cornwell, T. H. Gilfedder, A. Franzen, and I. Andonovic, "SLOB: a switch with large optical buffers for packet switching," J. Lightwave Technol., vol. 16, no. 10, pp. 1725-1736, Oct. 1998.

[4] H. Yang and S. J. B. Yoo, "A new optical switching fabric architecture incorporating rapidly switchable all-optical variable delay buffers," Proc. OFC2004, Los Angeles, USA, ThV2, Feb. 2004.

[5] Y. Yoo, J. Yu, and G. Chang, "A dynamically reconfigurable folded-path time delay buffer for optical packet switching," IEEE Photon. Technol. Lett., vol. 16, no. 11, pp. 2559-2561, Nov. 2004.

[6] R. Langenhorst, M. Eiselt, W. Pieper, G. Grobkopf, R. Ludwig, L. Kuller, E. Dietrich, and H. G. Weber, "Fiber loop optical buffer," J. Lightwave Technol., vol. 14, no. 3, pp. 324-335, March 1996. 
[7] H. Toda, F. Nakada, M. Suzuki, and A. Hasegawa, "An optical packet compressor based on a fiber delay loop," IEEE Photon. Technol. Lett., vol. 12, no. 6, pp. 708-710, June 2000.

\section{Introduction}

Optical packet switching (OPS) technology is the ultimate future-proof solution for next generation optical transport networks. It enables direct forwarding of data packets in the optical domain [1]. Optical buffers are key components in OPS systems that prevent packet collision along the same virtual path and wait for header processing time. The function of the optical buffer is, then, to store and release the data at a desired timing in optical format without conversion to the electrical format. Several approaches have been proposed to realize an optical buffer. Recently, innovative "slow light" all-optical buffer technologies using electro-magnetically induced transparency or photonic crystals have been investigated [2], but they remain at a development stage that is too primitive for communication applications. The only practical optical buffer is the fiber delay line. Depending on different topologies, four major types of optical buffer based on delay lines can provide variable buffer depths: cascaded $2 \times 2$ switches [3], star configurations $(1 \times \mathrm{M}$ switch) [4], tree topologies [5], and recirculating loops [6, 7]. Recirculating loop buffers are more compact and require few components to implement. However, recirculating loops can produce unexpected oscillation because of their configuration and optical amplifiers in the fiber ring, unless the fiber loop has a band-pass filter (BPF) with narrow bandwidth and with a center wavelength that is identical to the packet wavelength. For OPS nodes based on WDM techniques, optical buffers require wavelength-independent characteristics. Therefore, no oscillating recirculating loops are expected even if the bandwidth of the BPF in the loop is broader and the center wavelength does not match the signal wavelength.

To prevent unexpected oscillation, we use an optical frequency shifter in the fiber ring. This study demonstrates, experimentally, a tunable optical delay line based on a fiber ring constructed from an acousto-optic frequency shifter (AOS) and an erbium-doped fiber amplifier (EDFA). Comparison of the result of the case without AOS shows that the frequency shifter is effective for suppressing unexpected oscillation.

\section{Optical Tunable Delay Lines using a Fiber Ring}

\subsection{Configurations of the tunable delay line with a frequency shifter}

Tunable buffers include two functions: one is storing a packet in a fiber ring; another is selection from the fiber ring at favorite timing. Figure 1 (a) shows the configuration of the optical tunable delay line using a fiber ring with an AOS and EDFA. The storing function can be performed by the fiber ring 

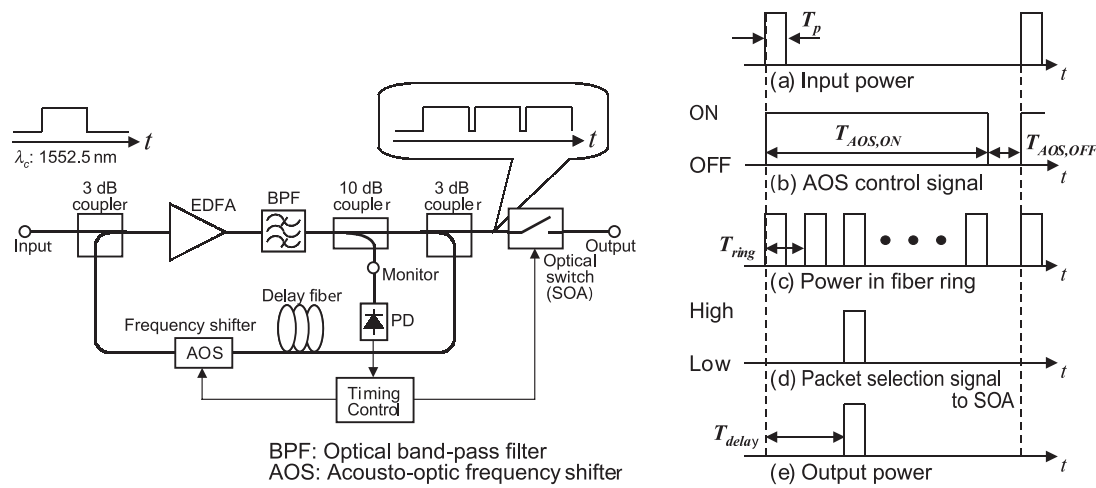

Fig. 1. Configuration of the optical tunable delay line using fiber ring with an acousto-optic frequency shifter and an EDFA, and a timing chart for controlling the delay lines.

with AOS and EDFA. The following semiconductor optical amplifier (SOA) plays the role of the second function of the delay-time selection. Figure 1 (b) shows the timing chart of the tunable delay line. The fiber length of the ring delays the packet when the optical packet signal pulse is incident to the fiber ring. The ring output is an optical pulse sequence constructed from replicas of the incident packet. If the time duration of the packet $T_{p}$ is shorter than the round trip time of the ring $T_{\text {ring }}$, then the optical switch following the ring selects the packet when the packet route is available.

A packet can experience many round trips in the fiber ring. Optical amplifiers, then, are necessary to compensate the optical loss of the ring. In our study, an EDFA is employed because of its high gain and low noise. The following band-pass filter (BPF) rejects amplified spontaneous emission (ASE) noise and residual pump light from the EDFA. An AOS is inserted into the ring to prevent oscillation from the ring cavity. If no AOS were used in the ring, the center frequency of the BPF would have to be identical to the signal wavelength; moreover, a quite narrow bandwidth of BPF is required. Those conditions limit the usable wavelength bandwidth of the buffer. In packetswitching nodes, many wavelengths come from the input port. Wavelengthindependent characteristics are adequate for tunable packet buffers. The AOS has the additional function of switching between the opening state and closing state of the fiber ring.

In our experiments, packet duration $T_{p}$ was $5.0 \mu \mathrm{s}$. The small signal gain and saturation output of EDFA were, respectively, $28.5 \mathrm{~dB}$ and $9.44 \mathrm{~mW}$. The noise of the EDFA was $7.0 \mathrm{~dB}$. The full-width at half-maximum (FWHM) of the BPF was $0.89 \mathrm{~nm}$. The center wavelength of the BPF was identical to the packet wavelength of $1552.5 \mathrm{~nm}$, which equals the standard WDM anchor wavelength. The delay fiber was a standard single-mode fiber with length of $1.08 \mathrm{~km}$. The frequency shift of AOS was $-120 \mathrm{MHz}$, much smaller than the EDFA gain bandwidth and FWHM of the BPF. The optical loss of the ring, except for the EDFA, was $17.7 \mathrm{~dB}$. An SOA was used as an optical switch to select a packet from the output pulse sequence. Timing control was achieved 
using a personal computer, but the PD monitor was not used for control in this experiment.

\subsection{Experimental results}

Figure 2 (a) shows the monitor port waveform of the fiber loop without AOS. A test packet was CW light sliced by an optical switch. The upper waveform is an input packet and the lower one shows the monitor port output. Power fluctuation is apparent at the monitor port output. It is caused by oscillation in the fiber loop at gain peak wavelength. The fiber ring cavity is unstable in the situation of no environmental control: it can induce the power fluctuation.

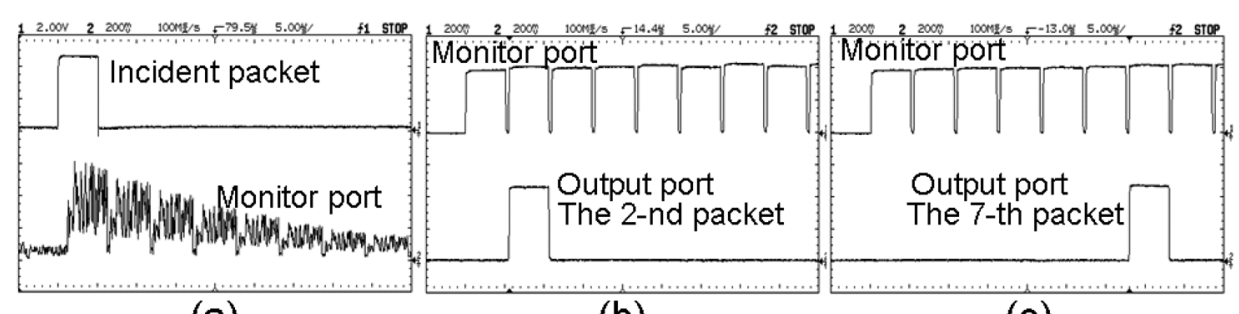

(a)

(b)

(c)

Fig. 2. Output packet envelope waveform: (a), without AOS; (b) and (c), with AOS; (b) selecting the second packet; (c) the seventh packet.

Figures 2 (b) and 2 (c) show the output waveform and that of the monitor port from the fiber delay lines with an AOS. Regarding Fig. 2 (b), ON timing of the optical switch was set to the second packet. Figure 2 (c) shows the case of the seventh packet. For the packet sequence from the monitor port, the packet replica shape shows no pulse shape degradation. For the packet from the output port, the output packet from the tunable delay line is well selected using an optical switch.

Figures 3 (b) and 3 (c) show the eye diagram of the second and seventh packets when the input packet was modulated by $2.5 \mathrm{Gbit} / \mathrm{s}$ pseudo-random data sequence (PN: 7 stages). The eye diagram in Fig. 3 (a) corresponds to the modulated input packet. It is apparent that the clear eye-height is maintained even if the packet passes through the fiber ring. In the eye diagram of delayed packets, there are two levels at the bottom of the eye diagram.

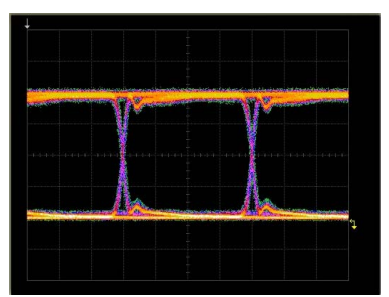

(a)

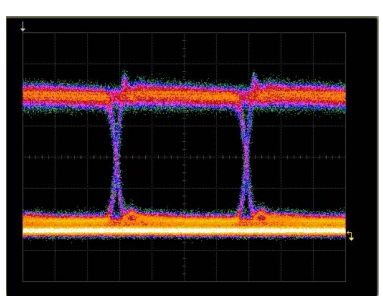

(b)

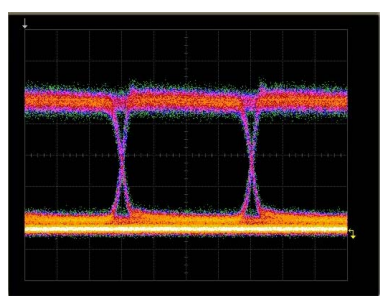

(c)

Fig. 3. Eye diagrams of $2.5 \mathrm{Gbit} / \mathrm{s}$ data packets: (a) input packet, (b) selecting the second packet, (c) the seventh packet.

(c) IEICE 2006

DOI: $10.1587 /$ elex.3.442

Received September 14, 2006

Accepted September 26, 2006

Published October 25, 2006 
Upper space line is dominated by ASE power from SOA in packet selection duration. The lowest line is corresponding to the output level without packet selection signal.

\section{Conclusion}

An optical tunable delay line using a fiber ring with an AO frequency shifter and an EDFA was demonstrated. Comparison to the case of no AOS reveals that the delayed packet has no power fluctuation attributable to unexpected oscillation. The packet replicas in the fiber ring have a uniform power envelope. The output packet from the tunable delay line is well selected using an optical switch. The eye height shows no degradation from the delay line of several-packet duration.

In recirculating loop buffers, other key issues are the upper limit of loop circulation and ASE accumulation. They will be clarified using SNR analysis with numerical simulation in a later paper, and will be compared with experimental results. 